\title{
Binary structures among large asteroids
}

\author{
M. Kaasalainen, J. Torppa, and J. Piironen \\ Observatory, University of Helsinki, PO Box 14, 00014 Helsinki, Finland \\ Received 19 November 2001 / Accepted 2 January 2002

\begin{abstract}
The imaging of well-observed large asteroids by lightcurve inversion has revealed objects, such as 44 Nysa and 41 Daphne, that are distinctly asymmetric and globally very different from equilibrium-like figures, indicating contact-binary structures. The shape of the Trojan asteroid 624 Hektor is very probably globally bifurcated. Together with the known large binary asteroids 216 Kleopatra, 90 Antiope, and 617 Patroclus, these findings indicate that a nonvanishing portion of large asteroids have binary structures.
\end{abstract}

Key words. solar system: minor planets, asteroids - techniques: photometric - methods: numerical

\section{Introduction}

Lightcurve inversion is an efficient deconvolution technique whose resolving capacity lies between space telescope and radar, and whose range extends from nearEarth to main-belt asteroids (Kaasalainen \& Torppa 2001; Kaasalainen et al. 2001; Kaasalainen 2001). The rotational state and the characteristic large-scale shape features of an object (such as those of 6489 Golevka or 433 Eros) can well be inferred from good-quality data by lightcurve inversion. The global shape of the target can usually be best described with a convex model as lightcurves seldom carry information on small shape details or nonconvex features.

Of order one hundred asteroids have been observed extensively enough for the reconstruction of a physical model. Photometric measurements of over 1000 asteroids (some of which have been observed for more than half a century) have been collected in the Uppsala Asteroid Photometric Catalogue (UAPC; Lagerkvist et al. 2001). We have used this catalogue as our data source, and we expect our ongoing survey to produce models of the global shapes, rotational states, and surface scattering characteristics of at least several tens of asteroids.

The UAPC survey has already revealed a variety of asteroid shapes (Kaasalainen et al. 2002). While small (kilometre-size) asteroids can exhibit fragment-like irregularities, most large (size over some $50 \mathrm{~km}$ ) asteroids we have analyzed can roughly be regarded as often considerable but quite random variations of quasiellipsoidal or quasi-equilibrium shapes. This is consistent

Send offprint requests to: M. Kaasalainen,

e-mail: Mikko.Kaasalainen@astro.helsinki.fi with the morphology and evolutionary histories expected of asteroidal bodies composed of predominantly homogeneous material. Some large asteroids, however, stand out from the majority. They display distinctly asymmetric shapes; even more remarkable, these asymmetries are of quite regular kind. Asteroids 44 Nysa and 41 Daphne, with IRAS size estimates, respectively, some $70 \mathrm{~km}$ and $180 \mathrm{~km}$ (Small Bodies Data Archives: http://pdssbn. astro.umd.edu/sbnhtml/index.html\# asteroids) are the foremost examples of such objects.

\section{Conical asymmetry}

We show the inversion model of the E-type asteroid 44 Nysa in Fig. 1. The three subfigures are shown at rotational phases $120^{\circ}$ apart, and the viewing geometry is equatorial. The light source and the observer are collocated, and the scattering model is chosen purely for illustrational purposes. The model is based on 63 lightcurves measured between 1949 and 1987, so the observations cover Nysa densely. Four typical lightcurves are shown in Fig. 2, together with the model fits. The shape model clearly represents a cone-like structure as the main feature of the global shape. The sides of the cone are probably concave: long, straight stretches on the convex solution usually indicate nonconvexity (cf. numerical Castalia-type simulations in Kaasalainen \& Torppa 2001 and the model of 433 Eros in Kaasalainen et al. 2001). The convex model is merely the most reliable representation available from photometric data. As is typical for lightcurve inversion, nonconvex models could neither improve the already very good lightcurve fit nor indicate any stable distinct nonconvex features: various models with concave "waists" gave as good a fit. This is especially characteristic of main-belt 

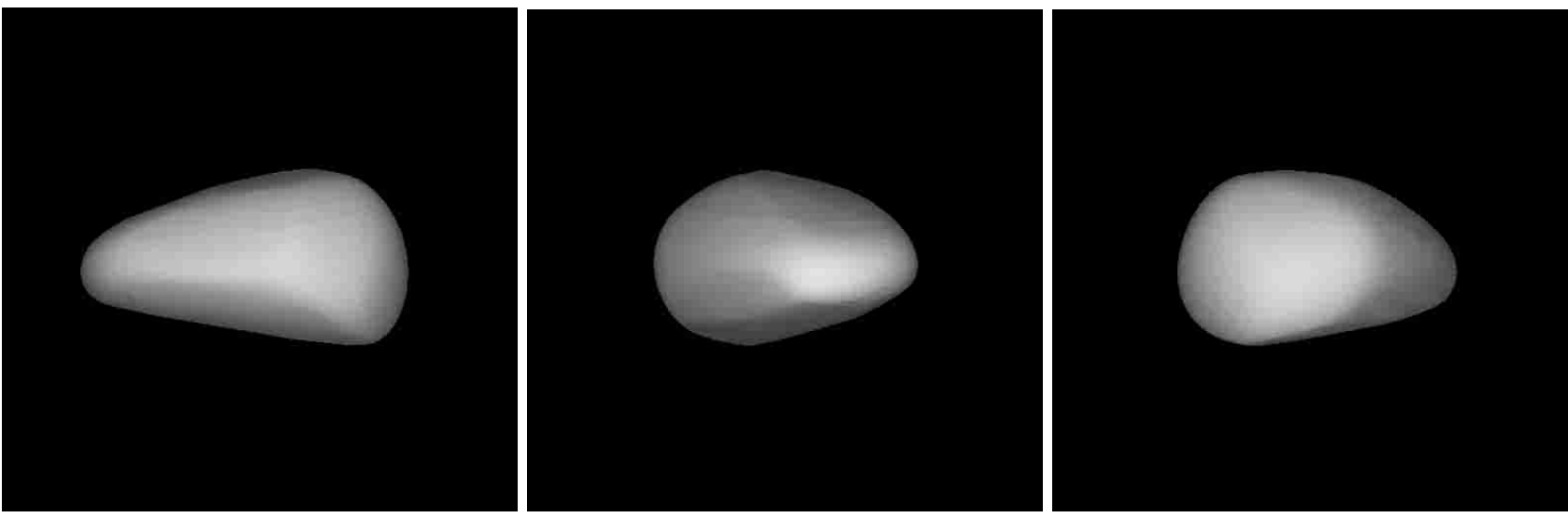

Fig. 1. Convex shape model of $44 \mathrm{Nysa}$, shown at rotational phases $120^{\circ}$ apart. The flat middle parts are probably concave in reality, implying a contact-binary structure.
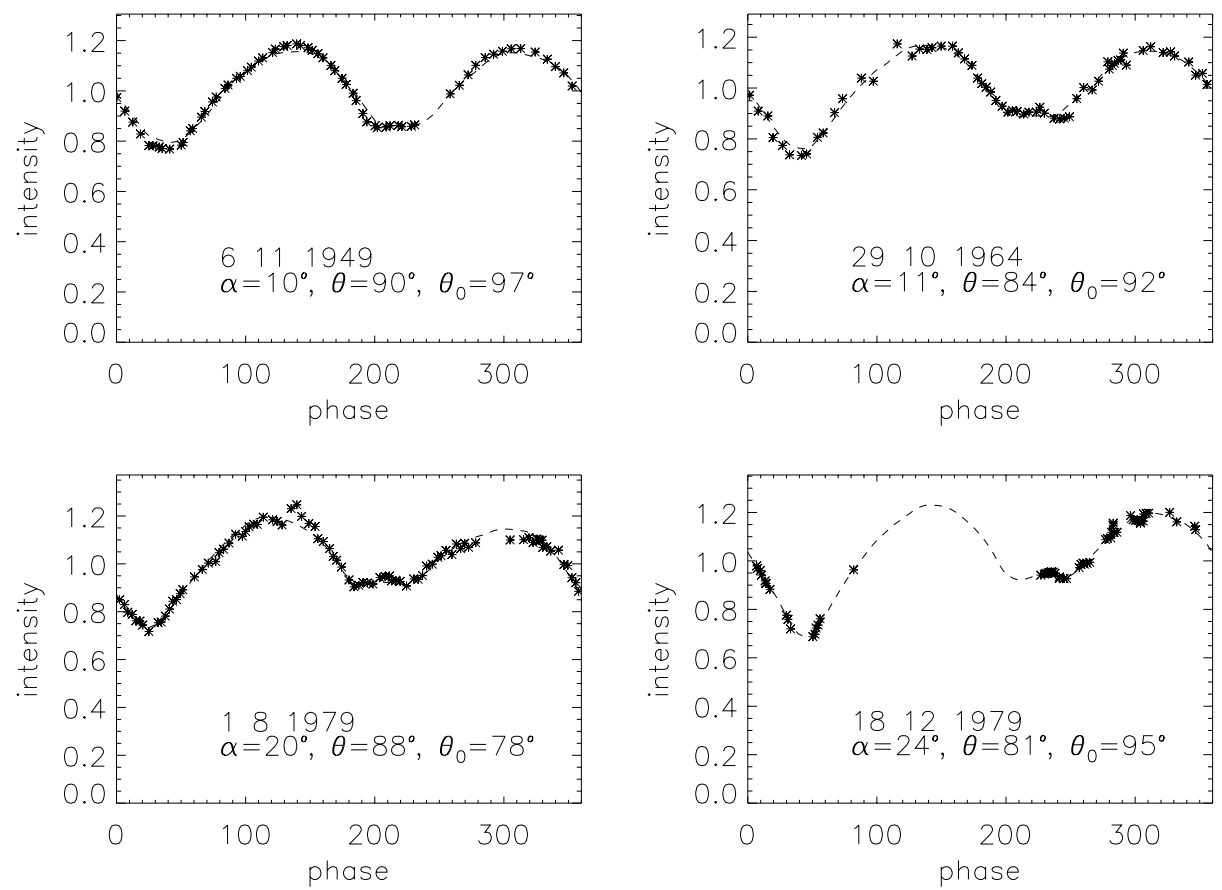

Fig. 2. Four lightcurves (asterisks) and the corresponding fits (dashed lines) for 44 Nysa. The rotational phase is given in degrees, and the brightness in units of relative intensity. The viewing latitude (measured from the North pole) is given by $\theta$, and the illumination latitude by $\theta_{0}$. The solar phase angle is given by $\alpha$.

asteroids, whose solar phase angles are seldom high enough for striking shadowing effects to be seen.

The lightcurves display the hallmarks of the cone shape: two minima at different levels, one blunt, the other sharp. The rms deviation of the lightcurve fits is only $0.02 \mathrm{mag}$, i.e., at the same level as the data noise. The solution was consistent with the scattering parameters quoted for Nysa in Bowell et al. (1989). A fortunate property of lightcurve inversion is that, when based more on the shapes of lightcurves than their absolute brightness values, the end result is not very sensitive to the chosen scattering model so long as it is reasonably realistic (Kaasalainen et al. 2001). For Nysa's period and pole direction we obtained $P=6.421417 \pm 0.000001 \mathrm{hrs}, \lambda=98^{\circ} \pm 2^{\circ}$, and $\beta=+58^{\circ} \pm 3^{\circ}$, where $\lambda$ and $\beta$ are, respectively, the ecliptic longitude and latitude. The surface albedo is quite uniform, and the adopted pole solution was clearly the best.

The cone shape of Nysa might be taken for a one-off curiosity, were it not for the fact that the asteroid 41 Daphne looks like its twin. We show the shape model of 41 Daphne in Fig. 3 in a sequence similar to Fig. 1. The model is based on 23 lightcurves measured between 1976 and 1988, when Daphne was well observed and illuminated from all directions. Four typical lightcurves of Daphne are shown in Fig. 4. Again, the fit deviation is only $0.02 \mathrm{mag}$. Daphne looks very much like Nysa, except that it is more irregular at its blunt end, as if a chip had been carved off. This end is also marked by a large area of higher surface albedo, 

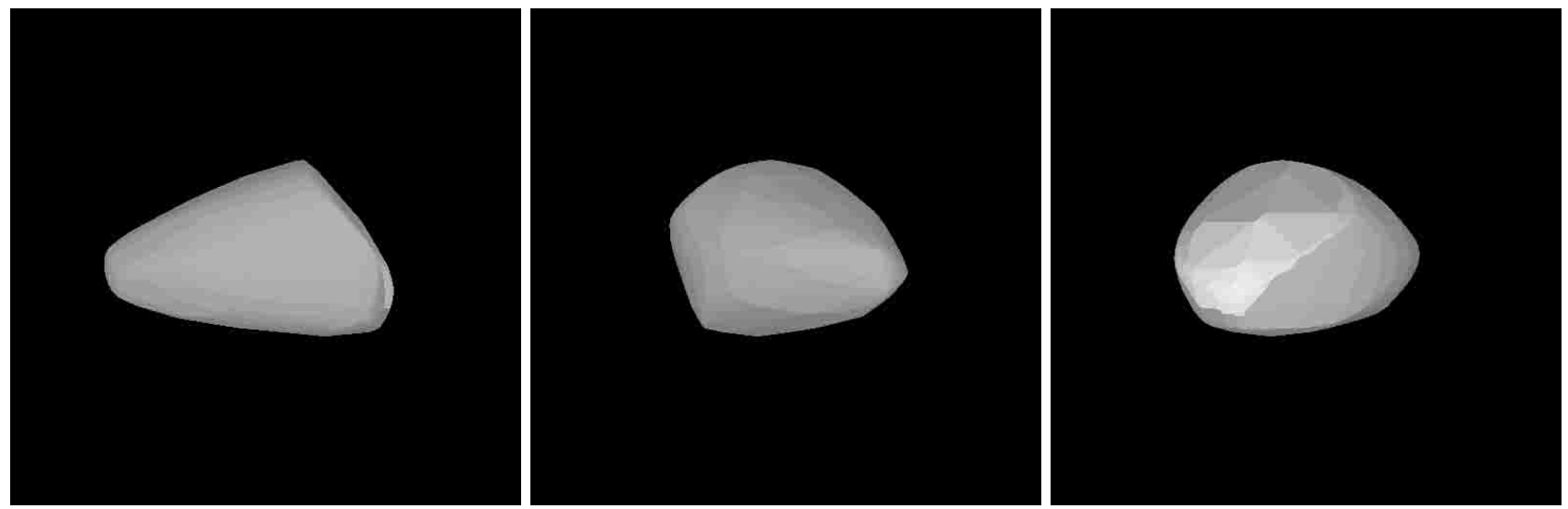

Fig. 3. Convex shape model of 41 Daphne, shown at rotational phases $120^{\circ}$ apart.
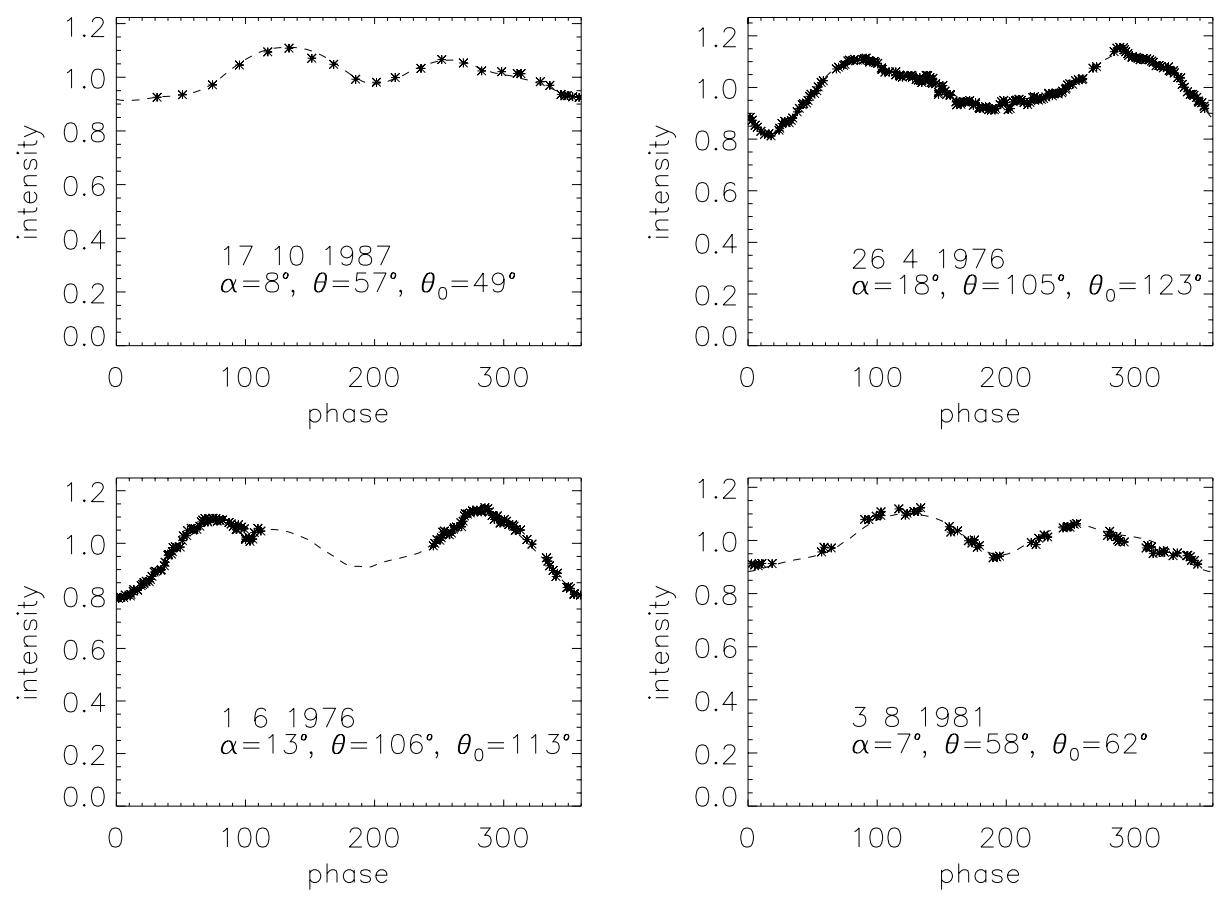

Fig. 4. Four lightcurves and the corresponding fits for 41 Daphne.

the brightest parts of which are some $30 \%-40 \%$ brighter than the rest of the surface. These features are perhaps suggestive of an impact.

The convex modelling procedure contains a strong indicator for testing whether there is significant albedo variegation on the surface or not (Kaasalainen \& Torppa 2001). Physically the most plausible solution (and mathematically a stable one) is, as corroborated by spacecraft images, to attribute lightcurve variation to shape as much as possible, and to invoke albedo variegation only when necessary. Daphne showed clear indication of moderate albedo variegation, so we modelled the albedo as a bright spot (Kaasalainen et al. 2002), using physically probable constraints. The spot was forced to spread smoothly over the blunt end, and various regularization strengths for this constraint always produced a similar-looking bright area in the "lower" part of the blunt end.

For Daphne's period and pole direction we obtained $P=5.987982 \pm 0.000003 \mathrm{hrs}, \lambda=196^{\circ} \pm 3^{\circ}$, and $\beta=$ $-31^{\circ} \pm 3^{\circ}$. This pole solution provided the best fit and the most consistent model (a "mirror" pole solution at $\lambda=345^{\circ}$ and roughly the same $\beta$ has a non-zero probability, but its shape solution is, in any case, qualitatively very similar). The scattering properties were completely consistent with those of an average C-class asteroid (Helfenstein \& Veverka 1989).

Of the some 40 asteroids we have analyzed so far, the lightcurves and the inversion result of the M-type asteroid 201 Penelope (roughly the same size as Nysa) also display asymmetric features similar to those of Daphne and Nysa, 
albeit in a very moderate fashion. Penelope could thus have structural similarities with the other two asteroids.

What is the physical structure of the asymmetric shapes? The cone-like shape would seem to be generic; for asteroids of this size, the most obvious explanation is a contact binary or a "compound asteroid" consisting of two components of unequal size, possibly covered with a deep rubble layer. The probably nonconvex sides of the cones are exactly what could be expected of contact binaries (cf. asteroid 4769 Castalia; Hudson \& Ostro 1994). Although equilibrium shapes of gravel-like material can vary considerably from those of fluids (Holsapple 2001), a conical rubble pile does not seem a plausible construction without larger solid components underneath to support it. A solid monolithic core is less probable as there would have to be a generic mechanism that first carves out such large regular asymmetric shapes and then protects them from impact disruption and deformations over long time scales. A congregation of debris would be more stable against such perturbative effects (Asphaug et al. 1998).

\section{Lightcurves of a bifurcated object}

A cone-like shape is the outcome of a contact structure whose components have different sizes, whereas equalsized loosely bound components produce "dumbbells" or "dogbones" such as 216 Kleopatra (Ostro et al. 2000). The D-type Trojan asteroid 624 Hektor (estimated size class some $150 \mathrm{~km}$ ) is most likely another symmetric double-lobed structure. Due to its distance from the Earth, Hektor's solar phase angles are always small (between $1^{\circ}$ and $7^{\circ}$ for our data set), so shadowing effects are never pronounced. Hence the inversion result (based on 19 lightcurves taken between 1957 and 1991) is necessarily much more qualitative than quantitative; nevertheless, it clearly betrays Hektor's character by its long, flat sides (separating two slightly rounded ends) whose most plausible interpretation is a globally nonconvex shape. This signature is actually stronger than the one in the similar model of Kleopatra. Conversely, the observed lightcurves (particularly their sharp minima) cannot be caused by an elongated but "round" body. Hektor is thus probably a very loose contact binary. Due to the low phase angles, a detailed model of the bifurcated shape cannot be obtained from photometric data alone (the fit already being very good again, within $0.03 \mathrm{mag}$ rms deviation).

To make sure that our shape interpretations are consistent, we created simulated lightcurves of various contactbinary shapes at observing geometries similar to those of Nysa, Daphne, and Hektor. While the simulated noise and the restricted solar phases prevented the recovery of nonconvex details, the reconstructed convex shapes always contained large flat regions around the "waist" of the contact structure. In a forthcoming paper, we will address this aspect of the inverse problem more closely and examine in detail exactly how much nonconvex information can be recovered in various observational circumstances (Durech \& Kaasalainen, in preparation).

\section{Discussion}

Binary configurations (contact or separate) are predicted and now known to be fairly common among small nearEarth asteroids (e.g., Bottke \& Melosh 1996; Benner et al. 2001; Mottola \& Lahulla 2000; Hudson \& Ostro 1994), but, as the radar model of 216 Kleopatra and the adaptive optics images of 90 Antiope (Merline et al. 2000) and 617 Patroclus (Merline et al. 2001) show, even large asteroids may be bifurcated or form binary systems. Our small sample set and inevitably biased data source hardly facilitate a detailed statistical analysis, but we can roughly estimate that, if the contact-binary hypothesis is valid, the fraction of asteroids of binary nature could well be at least a few per cent of the whole population of large (and intermediate-size) asteroids. Together with the increasing number of known binary near-Earth asteroids and the small satellites found accompanying large asteroids (e.g., Merline et al. 1999, 2000; Storrs et al. 2001; Brown \& Margot 2001), these findings indicate that we can well expect various non-single configurations to be common among asteroids in all size scales.

\section{References}

Asphaug, E., Ostro, S. J., Hudson, R. S., Scheeres, D. J., \& Benz, W. 1998, Nature, 393, 437

Benner, L. A. M., Nolan, M. C., Ostro, S. J., et al. 2001, IAU Circ., 7632/7730

Bottke, W. F., \& Melosh, H. J. 1996, Icarus, 124, 372

Bowell, E., Hapke, B., Domingue, D., et al. 1989, Application of photometric models to asteroids, in Asteroids II, ed. R. P. Binzel, T. Gehrels, \& M. S. Matthews (University of Arizona Press, Tucson), 524

Brown, M. E., \& Margot, J. L. 2001, IAU Circ., 7588

Helfenstein, P., \& Veverka, J. 1989, Physical characterization of asteroid surfaces from photometric analysis, in Asteroids II, ed. R. P. Binzel, T. Gehrels, \& M. S. Matthews (University of Arizona Press, Tucson), 557

Holsapple, K. 2001, Icarus, in press

Hudson, R. S., \& Ostro, S. J. 1994, Science, 263, 940

Kaasalainen, M., \& Torppa, J. 2001, Icarus, 153, 24

Kaasalainen, M., Torppa, J., \& Muinonen, K. 2001, Icarus, 153, 37

Kaasalainen, M. 2001, A\&A, 376, 302

Kaasalainen, M., Torppa, J., \& Piironen, J. 2002, Icarus, submitted

Lagerkvist, C.-I., Piironen, J., \& Erikson, A. 2001, Asteroid Photometric Catalogue, fifth update (Uppsala University Press, Uppsala)

Merline, W. J., Close, L. M., Dumas, C., et al. 1999, Nature, 401, 565

Merline, W. J., Close, L. M., Dumas, C., et al. 2000, BAAS, 32,1017

Merline, W. J., Close, L. M., Siegler, N., et al. 2001, IAU Circ., 7741

Mottola, S., \& Lahulla, F. 2000, Icarus, 146, 556

Ostro, S. J., Hudson, R. S., Nolan, M. C., et al. 2000, Science, 288,836

Storrs, A., Vilas, F., Landis, R., et al. 2001, IAU Circ., 7599 